\title{
Effects of Large Eddies on the Structure of the Marine Boundary Layer under Strong Wind Conditions
}

\author{
ISAAC GINIS \\ Graduate School of Oceanography, University of Rhode Island, Narragansett, Rhode Island \\ Alexander P. Khain and Elena Morozovsky \\ Institute of Earth Sciences, Hebrew University of Jerusalem, Jerusalem, Israel
}

(Manuscript received 13 August 2003, in final form 22 June 2004)

\begin{abstract}
A model of the atmospheric boundary layer (BL) is presented that explicitly calculates a two-way interaction of the background flow and convective motions. The model is utilized for investigation of the formation of large eddies (roll vortices) and their effects on the structure of the marine boundary layer under conditions resembling those of tropical cyclones. It is shown that two main factors controlling the formation of large eddies are the magnitude of the background wind speed and air humidity, determining the cloud formation and latent heat release. When the wind speed is high enough, a strong vertical wind shear develops in the lower part of the $\mathrm{BL}$, which triggers turbulent mixing and the formation of a mixed layer. As a result, the vertical profiles of velocity, potential temperature, and mixing ratio in the background flow are modified to allow for the development of large eddies via dynamic instability. Latent heat release in clouds was found to be the major energy source of large eddies. The cloud formation depends on the magnitude of air humidity.

The most important manifestation of the effects of large eddies is a significant increase of the near-surface wind speed and evaporation from the sea surface. For strong wind conditions, the increase of the near-surface speed can exceed $10 \mathrm{~m} \mathrm{~s}^{-1}$ and evaporation from the sea surface can double. These results demonstrate an important role large eddies play in the formation of BL structure in high wind speeds. Inclusion of these effects in the BL parameterizations of tropical cyclone models may potentially lead to substantial improvements in the prediction of storm intensity.
\end{abstract}

\section{Introduction}

Theory and numerical simulations amply demonstrate the sensitivity of tropical cyclone (TC) intensity to sea surface fluxes of momentum and enthalpy in the storm core (e.g., Emanuel 1995; Kurihara and Tuleya 1974; Tuleya 1994). The surface fluxes are in turn directly related to the vertical transfer of momentum, heat, and humidity in the atmospheric boundary layer (BL). Yet very little is known about air-sea exchanges and vertical transfer processes in extremely high wind speeds. In tropical cyclone research and forecast models, the description of the BL is typically based on local gradients of wind and potential temperature and vertical diffusivity coefficients (e.g., Kurihara et al. 1998; Bao et al. 2000). In the commonly used $K$ theory, the turbulence is represented by an eddy-diffusivity coefficient, $K$, which is typically parameterized as a function of the local Richardson number. While $K$-theory-based param-

Corresponding author address: Dr. Isaac Ginis, Graduate School of Oceanography, University of Rhode Island, Narragansett, RI 02882.

E-mail: iginis@gso.uri.edu eterizations are computationally efficient and produce reasonable results, in many cases they have some significant limitations, as pointed out by many previous studies (e.g., Deardorff 1972; Holtslag and Moeng 1991; Khain et al. 1986). The most fundamental criticism is $K$ theory does not account for transfer of heat, moisture, and momentum caused by "large eddies," which are known to be a very common feature of the atmospheric boundary layer. These eddies have a characteristic spatial scale from a few hundred meters to a few kilometers (LeMone 1973, 1976; Etling and Brown 1993). Under certain conditions (Emanuel 1994; Glendening 2000) large eddies are realized in the form of "roll vortices," helical circulation patterns that typically have vertical scales of the same order as the depth of the BL. Rolls have been observed by radar, aircraft, and surface station observations in cold-air outbreaks and sea breezes or in the lee of coastal mountains and simulated by highresolution models (e.g., Brown 1974; Atlas et al. 1986; Liu and Kogan 1998). They can also arise because of daytime heating of the continental clear-air convective boundary layer. Rolls are regularly identified by coherent structures of cloudiness, or cloud "streets," elon- 


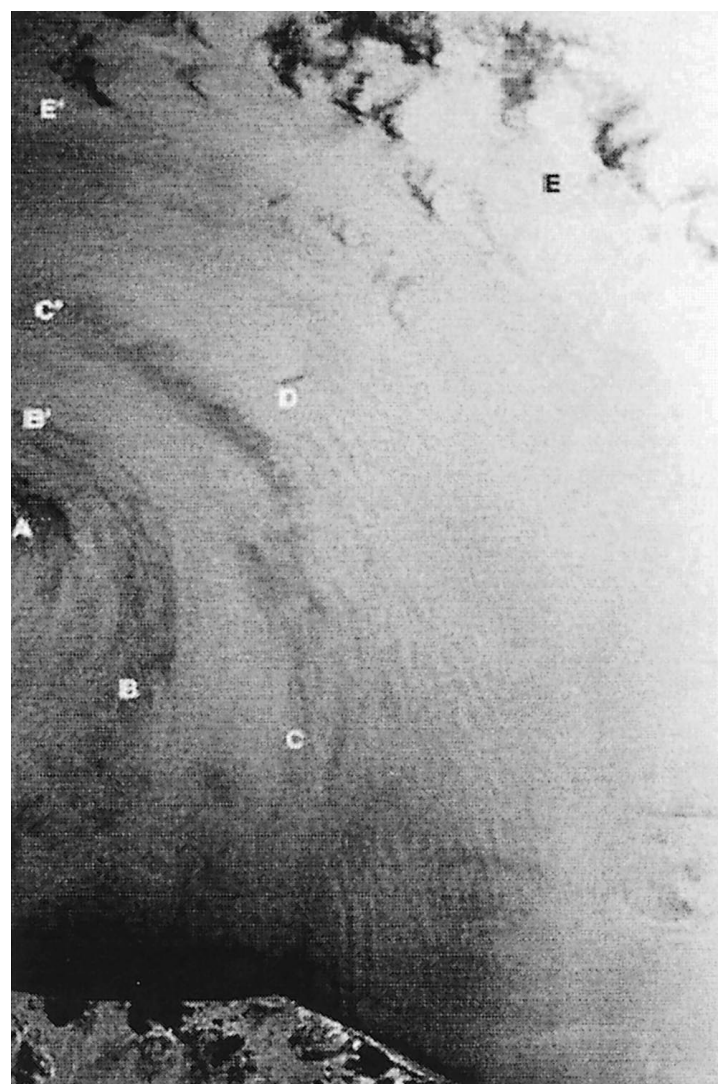

FIG. 1. Roll vortices in the boundary layer of a hurricane: $R A$ $D A R S A T$ SAR image of the sea surface roughness during Hurricane Mitch on 27 Oct 1998. A first rainband $30 \mathrm{~km}$ east of the center is seen in the western edge of the image, $\mathrm{A}$, and a stratiform rainband is seen along $\mathrm{B}-\mathrm{B}^{\prime}$ about $40-60 \mathrm{~km}$ east of the center. A third, more convective band, $\mathrm{C}-\mathrm{C}^{\prime}$, occurs about $70-120 \mathrm{~km}$ northeast of the center. Swan Island (Islas Santanilla) is located at D. Strongly convective outer bands are noted along E-E'. Secondary circulations in the regions between the main rainbands with wavelengths of 4-6 km are clearly visible. Adopted from Katsaros et al. (2000).

gated closely along the mean wind direction in the $\mathrm{BL}$ or at a small angle to it.

Recent observations have revealed roll vortices in the BL of tropical cyclones. Wurman and Winslow (1998) observed rolls with horizontal scale of about $600 \mathrm{~m}$ in the core region of landfalling tropical cyclones using high-resolution Doppler radars. Morrison et al. (2002) observed roll vortices in Doppler radar data from Hurricanes Fran, Bonnie, and Georges in 1998 with wavelengths of about $1.5 \mathrm{~km}$ and roll orientation of approximately $10^{\circ}$ from the surface wind direction. Similarly, Katsaros et al. (2002) discovered periodic secondary circulations in the regions between the main rainbands of Hurricanes Bonnie, Danielle, Georges, and Mitch in 1998 in the analysis of RADARSAT synthetic aperture radar (SAR) images of the sea surface roughness (Fig. 1). These findings have been the primary motivation of the present study. We speculate that large eddies are a ubiquitous feature of the BL in tropical cyclones. Fur-
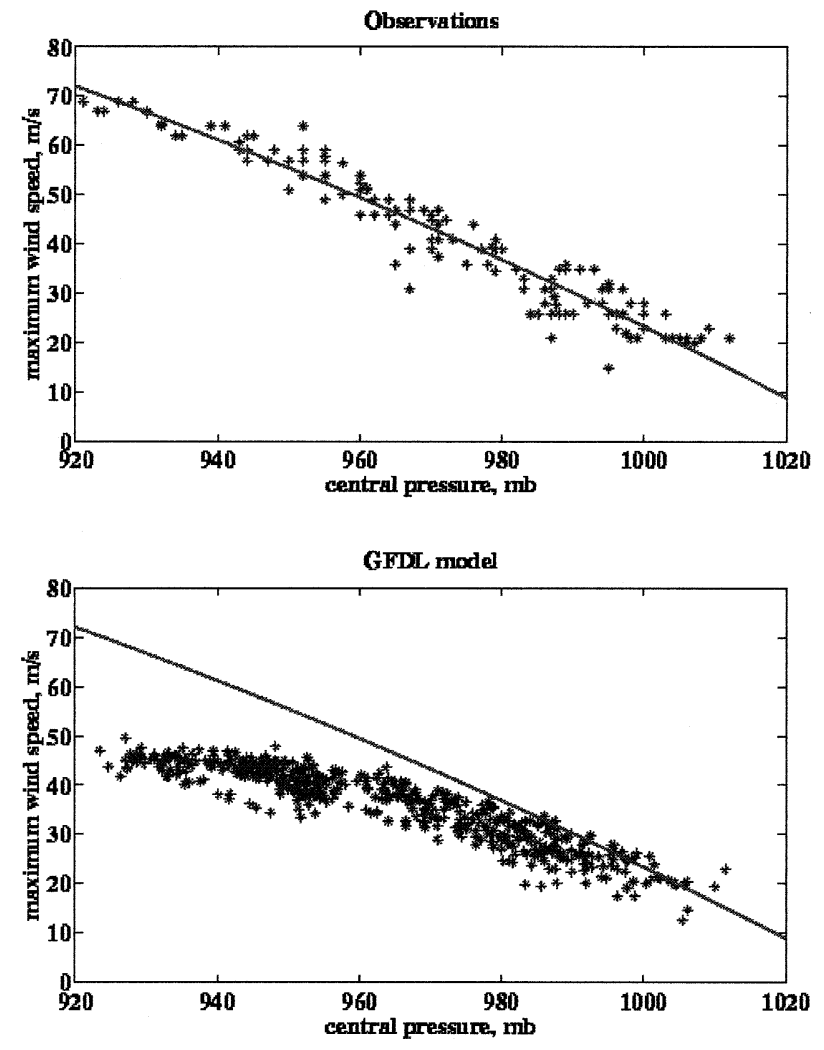

FIG. 2. Wind-pressure relationship in (top) the observations and (bottom) the GFDL/URI coupled hurricane-ocean model during the 1999 hurricane season.

thermore, it is suggested that they can contribute significantly to the vertical transfer of momentum, heat, and moisture, as well as the air-sea fluxes, as pointed out by previous studies.

Lack of adequate consideration of the large eddy effects in the BL may be one of the reasons for the limited tropical cyclone intensity forecast skill by hurricane prediction models. Recently implemented to operations, the Geophysical Fluid Dynamics Laboratory (GFDL)/University of Rhode Island (URI) coupled hurricane-ocean model helped to improve the intensity predictions measured by the central pressure (Bender and Ginis 2000). However, it has not always translated into improvements in predicting the maximum wind speed. This is mainly due to underestimations of the surface winds in strong tropical cyclones. This is illustrated in Fig. 2 through comparisons of wind-pressure relationships derived from observations and the GFDL/URI model. In strong wind conditions, the GFDL/URI model tends to underpredict surface wind speeds for a given central pressure and thus exhibits a large negative bias. This limited skill may be attributed to an insufficient, $1 / 6^{\circ}$, horizontal grid resolution. However, the more likely reason is inadequate representation of the physical processes, in particular not considering the contribution of large eddies in the modeling of the BL and air-sea fluxes. 
Although spatial resolution of mesoscale atmospheric models has increased in recent years with increased computer power, it still remains too coarse to resolve individual large eddies (hereafter, LE) in the BL. Large eddies, similar to small-scale turbulence, remain subgrid scale in these models and therefore have to be parameterized. Subgrid parameterizations are usually based on 1) semiempirical relationships between moments of different order (Garratt 1992), 2) derivations from large eddy simulation (LES) models (Pope 2000), or 3) empirical analyses of observations (Hong and Pan 1996). These parameterizations are typically obtained in low and moderate wind speeds. To our knowledge, the effect of large eddies on the BL structure has never been studied in TC models.

Our main objective in this study is to investigate possible mechanisms leading to the formation of large eddies under conditions resembling those in tropical cyclones and to assess their effects on the vertical transport of momentum and enthalpy and the BL structure using a high-resolution, nonhydrostatic BL model. In previous observational and modeling studies, large eddies were investigated in typically weak and moderate wind conditions and/or a thermally unstable BL where it is heated from below or cooled from above (LeMone 1973, 1976; Etling and Brown 1993). The BL in a tropical cyclone has at least two distinct features. First, it is typically characterized by strong winds and vertical wind shears. Second, stratification is often stable in the zones of descending air at the TC periphery and in the zones of decreased SST caused by the TC-ocean interaction. These features are emphasized in this study.

The paper is organized as follows. General approach is presented in section 2. A brief description of the model is given in section 3 . The results of numerical simulations are discussed in section 4. Finally, a summary is presented in section 5 .

\section{General approach}

The equations for atmospheric motions in convectivescale models are often written for deviations from either the "reference" or "mean" values. In the former approach (used, e.g., in the classical Rayleigh-Bernard problem) all variables are divided onto the initial (reference values) and their deviations. In this approach the averaged (e.g., in the horizontal direction) values of the deviations are not equal to zero, In the latter approach, which is widely used in most numerical models and in this study, any spatial and time-dependent variable $a(\mathbf{x}$, $t)$ is represented as $a=\bar{a}(\mathbf{x}, t)+a^{\prime}(\mathbf{x}, t)$, where $\bar{a}(\mathbf{x}$, $t)$ is the mean variable that is obtained by averaging of the initial equations with respect to time and space. If the time and spatial scales of averaging are much larger than the scales of the deviations then $\overline{a^{\prime}}=0$. The averaged equations contain terms representing moments of second order (e.g., $\overline{a^{\prime} w^{\prime}}$ ), which are expressed through the mean variables using a closure procedure (parameterization). The time and spatial scales of averaging determine the type of unresolved motions described by the deviations. If, for instance, the scale of spatial averaging is less than $\sim 100 \mathrm{~m}$, the deviations represent small-scale turbulence, which is usually parameterized using local $K$ theory. The equations of most general circulation models (GCMs) and regional models are typically derived by averaging over a spatial scale exceeding several kilometers (or tens of kilometers). In this case the deviations describe both small-scale turbulence and convective motions (large eddies).

It is well recognized that the contribution of large eddies to the vertical fluxes of momentum, temperature, and humidity is very important in the BL. Large eddies can be simulated explicitly by 3D LES models (e.g., Soong and Ogura 1980; Moeng 1984; Glendening 2000). Spatial resolution of these models is several tens of meters in all directions. Presently, computer limitations do not allow using LES models for simulation of large-scale and mesoscale phenomena (e.g., tropical cyclones), hence the necessity for parameterization of large eddies arises. Since the vertical scales of LE are of the same order as the depth of the BL, the local $K$ theory is not valid any longer. In some models highorder closure schemes are applied for large eddy parameterization (e.g., Mellor and Yamada 1982; Moeng and Wyngaard 1989). However, LEs are determined by different dynamic and thermodynamic processes in the BL, including the formation of clouds accompanied by heating due to condensation and cooling due to evaporation. It makes the LE parameterization problem especially difficult and thus inspires the search for new methods for description of large eddies in GCM and regional models.

In this paper we present such a new method. Based on previous observational and theoretical studies we assume that the preferable structure of large eddies in the BL at strong winds is roll vortices (or cloud streets in the cloudy BL) elongated along the mean flow. This assumption allows neglecting the derivatives of all deviations in the along-axis direction of roll vortices and splitting the full 3D system of equations into two coupled 2D subsystems (see next section for details). One subsystem describes nonhydrostatic equations that explicitly resolve large eddies. It is coupled with another subsystem of hydrostatic equations that describes the evolution of the mean flow. Such splitting is based on the fact that the length and time scales characterizing the mean flow and large eddies are very different. Khain et al. (1986) successfully used this approach for investigation of large eddies in the BL in trade winds. Here it is applied for conditions typical of the BL in tropical cyclones.

Our approach to the parameterization of large eddies in the BL resembles a recently emerging approach called "superparameterization" (Grabowski 2001; Randall et al. 2003). Superparameterization consists of a cloudresolving two-dimensional system embedded into a 


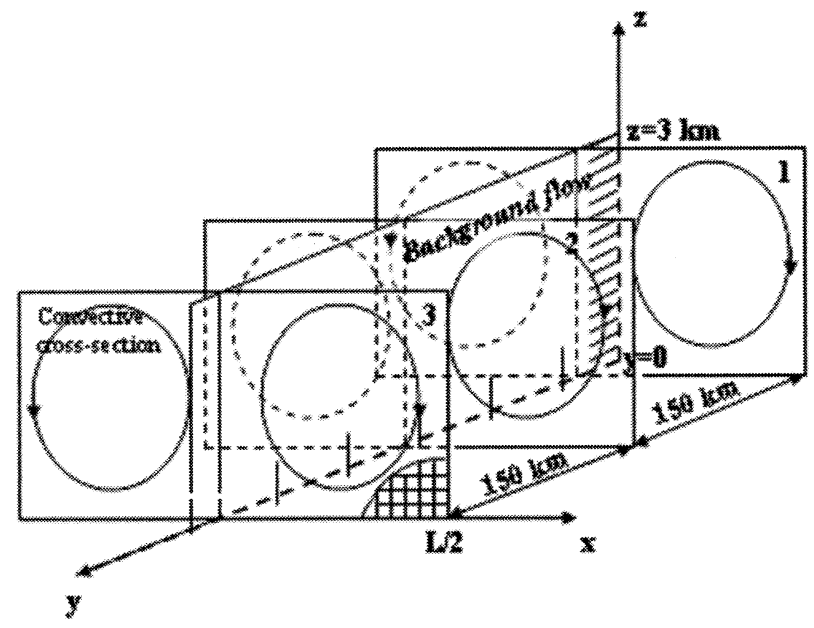

FIG. 3. Geometry of the finite-grid structure of the BL model.

GCM, allowing explicit cloud simulations. Similarly, our method can be called superparameterization of the BL, which includes a large eddy resolving two-dimensional system coupled with a system representing the large-scale mean flow. We show below how the coupled equation systems should be properly derived to describe the interaction between the convective processes, represented by large eddies, and the large-scale processes.

We use a simplified geometry in this study, shown in Fig. 3, in which the $y$ axis is directed along the mean flow, the $x$ axis is perpendicular to it, and the $z$ axis is in the vertical direction. We consider the atmospheric marine boundary layer of a depth $H$ in the Cartesian coordinates. We assume that (a) large eddies are realized in the form of roll vortices extended along the direction of the background wind, and (b) the spatial scale of the roll vortices along the $x$ axis and in the vertical is much smaller that the spatial scale along the $y$ axis.

The structure of the BL shown in Fig. 3 is, of course, very idealized. In reality, the roll structure is not strictly periodic, and roll vortices are not invariant in the alongaxis direction. The length of roll vortices varies and can reach several tens of kilometers, while the distance between neighboring rolls can vary from hundreds of meters to a few kilometers. According to the modeling results of Glendening (2000) using an LES model, the characteristic along-axis length is about 5 times of that in the cross-axis direction. The BL conditions used in that study could have led to some underestimation of this ratio. However, even the ratio $\sim 5: 1$ supports our assumption that the derivatives of the convective motions along the mean flow are significantly smaller than across the flow.

It should also be noted that the spatial structure of the rolls shown in Fig. 3 does not imply that the lengths of roll vortices are of the same scale as the distance between the convective cross sections (in our case 150 $\mathrm{km}$ ). Any convective parameterization (it is the main concept of the parameterizability) is based on the as- sumption that the statistical characteristics of subgridscale motions (in our case, the convective motions) are fully determined by the large-scale environment. In each cross section the convective motions have the properties corresponding to the large-scale forcing within the area of the cross section. Therefore, the roll vortices formed in different convective cross sections do not necessarily have to belong to the same rolls. The assumption that large eddies are realized in the form of roll vortices allows us to neglect the derivatives of the convective variables along the mean flow direction and thus to reduce the complex $3 \mathrm{D}$ problem to much simpler 2D problems.

\section{The boundary layer model}

\section{a. Governing equations}

The splitting of the full 3D system of equations is done by averaging the equations along the $x$ axis using the following operator:

$$
\bar{a}=1 / L \int_{0}^{L} a d x,
$$

where $L$ is the width of two or several rolls. Each dependent variable is represented as a sum

$$
a=\bar{a}+a^{\prime} .
$$

The averaged values, denoted as $\bar{a}$, will be referred to as mean (background) flow variables; the perturbations, denoted by $a^{\prime}$, will be referred to as convective-scale (or simply convective) variables. According to (1), an averaged perturbation is equal to zero. As an example of the splitting procedure, we apply (1)-(2) to the continuity equation written as $\operatorname{div} \mathbf{V}=0$, where $\mathbf{V}=(u$, $v, w)$ is the velocity vector. Assuming periodic boundary conditions along the $x$ axis, we can obtain two 2D continuity equations - one for the background flow velocity components in the $y-z$ plane and one for the velocity perturbations in the $x-z$ plane:

$$
\begin{gathered}
\frac{\partial \bar{v}}{\partial y}+\frac{\partial \bar{w}}{\partial z}=0, \\
\frac{\partial u^{\prime}}{\partial x}+\frac{\partial w^{\prime}}{\partial z}=0 .
\end{gathered}
$$

In Eq. (3) $\bar{v}$ and $\bar{w}$ are the horizontal and vertical components of the background flow velocity. In the derivation of Eq. (4) it is assumed that $\partial v^{\prime} / \partial y \ll \partial u^{\prime} / \partial x$. This assumption is valid because $v^{\prime}$ and $u^{\prime}$ have similar order of magnitudes but the horizontal scale in the $y$ direction is significantly larger than the horizontal scale in the $x$ direction. Applying (1) and (2) to the governing 3D system of equations (not shown; see Khain and Ingel 1988, 1995 for details), we derive the following equations describing conservation of momentum and heat in the background flow: 


$$
\begin{aligned}
& \frac{\partial \bar{v}}{\partial t}+\bar{v} \frac{\partial \bar{v}}{\partial y}+\bar{w} \frac{\partial \bar{v}}{\partial z}=-\frac{1}{\rho} \frac{\partial \bar{P}}{\partial y}-\frac{\partial \overline{w^{\prime} v^{\prime}}}{\partial z}+\bar{T}_{v}, \\
& \frac{\partial \bar{\theta}}{\partial t}+\bar{v} \frac{\partial \bar{\theta}}{\partial y}+\bar{w} \frac{\partial \bar{\theta}}{\partial z}=\frac{L}{c_{p}} \bar{\phi}-\frac{\partial \overline{w^{\prime} \theta^{\prime}}}{\partial z}+\bar{T}_{\theta},
\end{aligned}
$$

where $\bar{\theta}$ is potential temperature, and $\bar{\phi}$ is averaged rate of heating/cooling due to phase transition and radiation. Terms $\bar{T}_{v}$ and $\bar{T}_{\theta}$ describe the contribution of small-scale turbulent mixing (diffusion) of momentum and heat, correspondingly. Terms $-\left(\partial \overline{w^{\prime} v^{\prime}} / \partial z\right)$ and $-\left(\partial \overline{w^{\prime} \theta^{\prime}} / \partial z\right)$ describe the contribution of convective-scale motions (large eddies). Since we use an idealized background flow and the Coriolis force does not influence the structure of large eddies because of their small spatial scale, we neglect all Coriolis force effects in this study for simplicity. The complete system of equations (not shown) also includes the equations for mixing ratio, $\bar{q}$, and averaged cloud water content (CWC), $\bar{l}$, which have similar structures as Eq. (6) and the static equation.

The equations that govern the convective-scale motions are obtained by subtracting the equations for the averaged values from the full system of equations. As with the continuity equation, we neglect the derivatives of the perturbations along the $y$ axis and thus reduce the $3 \mathrm{D}$ equations to $2 \mathrm{D}$ equations written in $x-z$ coordinate plane. For example, the equation for convective velocity component $v^{\prime}$ is written as follows:

$$
\begin{aligned}
\frac{\partial v^{\prime}}{\partial t} & +u^{\prime} \frac{\partial v^{\prime}}{\partial x}+w^{\prime} \frac{\partial v^{\prime}}{\partial z} \\
& =\frac{\partial \overline{w^{\prime} v^{\prime}}}{\partial z}-\bar{w} \frac{\partial v^{\prime}}{\partial z}-w^{\prime} \frac{\partial \bar{v}}{\partial z}-v^{\prime} \frac{\partial \bar{v}}{\partial y}+T_{v}^{\prime} .
\end{aligned}
$$

The equations for convective velocity components $u^{\prime}$ and $w^{\prime}$ are written in terms of the perturbations of vorticity $\eta^{\prime}=\partial w^{\prime} / \partial x-\partial u^{\prime} / \partial z$ and streamfunction $\psi^{\prime}$ $\left[u^{\prime}=-\left(\partial \psi^{\prime} / \partial z\right), w^{\prime}=\partial \psi^{\prime} / \partial x\right]$ :

$$
\begin{aligned}
\frac{\partial \eta^{\prime}}{\partial t}+u^{\prime} \frac{\partial \eta^{\prime}}{\partial x}+w^{\prime} \frac{\partial \eta^{\prime}}{\partial z}= & g \frac{\partial}{\partial x}\left(\frac{\theta^{\prime}}{\theta}+0.61 q^{\prime}-l^{\prime}\right) \\
& -\frac{\partial \bar{w} \eta^{\prime}}{\partial z}+\frac{\partial \overline{w^{\prime} \eta^{\prime}}}{\partial z}+T_{\eta}^{\prime}, \\
\eta^{\prime}= & \frac{\partial^{2} \psi^{\prime}}{\partial x^{2}}+\frac{\partial^{2} \psi^{\prime}}{\partial z^{2}} .
\end{aligned}
$$

The equation for the perturbations of potential temperature is written as follows:

$$
\begin{aligned}
\frac{\partial \theta^{\prime}}{\partial t} & +u^{\prime} \frac{\partial \theta^{\prime}}{\partial x}+w^{\prime} \frac{\partial \theta^{\prime}}{\partial z} \\
& =\frac{L}{c_{p}} \phi^{\prime}+\frac{\partial \overline{w^{\prime} \theta^{\prime}}}{\partial z}-\bar{w} \frac{\partial \theta^{\prime}}{\partial z}-w^{\prime} \frac{\partial \bar{\theta}}{\partial z}+T_{\theta}^{\prime} .
\end{aligned}
$$

Similar equations are written for $q^{\prime}$ and $l^{\prime}$. Terms $T_{v}^{\prime}$, $T_{\eta}^{\prime}$, and $T_{\theta}^{\prime}$ in the above equations describe the smallscale turbulent fluxes. Equations (7)-(10) include terms that represent the influence of the background flow on the perturbations, such as $\bar{w}\left(\partial \eta^{\prime} / \partial z\right), \bar{w}\left(\partial \theta^{\prime} / \partial z\right), w^{\prime}(\partial \bar{v} /$ $\partial z)$, and $w^{\prime}(\partial \bar{\theta} / \partial z)$. The last two terms are of special importance since they describe advection of the background values by the convective-scale vertical velocity. Note that Eqs. (7)-(10) are similar to corresponding equations in LES models written in terms of perturbations from the mean values (e.g., Soong and Ogura 1980). In our case, however, these equations are solved together with the time-dependent background flow equations in a fully coupled mode.

The small-scale turbulent fluxes (we consider $T_{v}$ as an example) are parameterized as follows:

$$
T_{v}=\frac{\partial}{\partial z}\left(k_{z} \frac{\partial v}{\partial z}\right)+\frac{\partial}{\partial x}\left(k_{x} \frac{\partial v}{\partial z}\right)+\frac{\partial}{\partial y}\left(k_{y} \frac{\partial v}{\partial y}\right),
$$

where $k_{x}, k_{y}, k_{z}$ are the small-scale turbulent diffusion coefficients in the respective directions. Applying the averaging operators (1) and (2) gives

$$
\begin{aligned}
\bar{T}_{v}= & \frac{\partial}{\partial z}\left(\bar{k}_{z} \frac{\partial \bar{v}}{\partial z}\right)+\frac{\partial}{\partial z} \overline{\left(k_{z}^{\prime} \frac{\partial v^{\prime}}{\partial z}\right)}+\frac{\partial}{\partial x}\left(\bar{k}_{x} \frac{\partial \bar{v}}{\partial x}\right) \\
& +\frac{\partial}{\partial x} \overline{\left(k_{x}^{\prime} \frac{\partial v^{\prime}}{\partial x}\right)}+\frac{\partial}{\partial y}\left(\bar{k}_{y} \frac{\partial \bar{v}}{\partial y}\right)+\frac{\partial}{\partial y} \overline{\left(k_{y}^{\prime} \frac{\partial v^{\prime}}{\partial y}\right)} .
\end{aligned}
$$

Omitting derivatives of the averaged values with respect to $x$ (the values averaged along $x$ axis do not depend on $x$ ) and term $(\partial / \partial y) \overline{\left[k_{y}^{\prime}\left(\partial v^{\prime} / \partial y\right)\right]}$ containing negligibly small derivative $\partial v^{\prime} / \partial y$, we obtain

$$
\bar{T}_{v}=\frac{\partial}{\partial z}\left(\bar{k}_{z} \frac{\partial \bar{v}}{\partial z}\right)+\frac{\partial}{\partial z} \overline{\left(k_{z}^{\prime} \frac{\partial v^{\prime}}{\partial z}\right)}+\frac{\partial}{\partial y}\left(\bar{k}_{y} \frac{\partial \bar{v}}{\partial y}\right) .
$$

Assuming that $\bar{k}_{y}=k_{H}$ is constant (it is set to be equal to $10^{3} \mathrm{~m}^{2} \mathrm{~s}^{-1}$ in our calculations), we have finally

$$
\bar{T}_{v}=\frac{\partial}{\partial z}\left(\bar{k}_{z} \frac{\partial \bar{v}}{\partial z}\right)+\frac{\partial}{\partial z} \overline{\left(k_{z}^{\prime} \frac{\partial v^{\prime}}{\partial z}\right)}+k_{H} \frac{\partial^{2} \bar{v}}{\partial y^{2}} .
$$

Note that in the numerical experiments discussed below the horizontal diffusion terms are significantly smaller than the advection terms and thus do not play any important role (except some smoothing of the flow in the horizontal direction).

Subtracting Eq. (14) from Eq. (11) and neglecting the derivatives of the perturbations with respect to $y$ and the derivatives of the averaged values with respect to $x, T_{v}^{\prime}$ can be written as:

$$
\begin{aligned}
T_{v}^{\prime}= & \frac{\partial}{\partial z}\left(k_{z}^{\prime} \frac{\partial v^{\prime}}{\partial z}\right)+\frac{\partial}{\partial z}\left(\bar{k}_{z} \frac{\partial v^{\prime}}{\partial z}\right)+\frac{\partial}{\partial z}\left(k_{z}^{\prime} \frac{\partial \bar{v}}{\partial z}\right) \\
& -\frac{\partial}{\partial z} \overline{\left(k_{z}^{\prime} \frac{\partial v^{\prime}}{\partial z}\right)}+\frac{\partial}{\partial x}\left(k_{x}^{\prime} \frac{\partial v^{\prime}}{\partial x}\right)+\frac{\partial}{\partial x}\left(\bar{k}_{x} \frac{\partial v^{\prime}}{\partial x}\right) \\
& +\frac{\partial}{\partial y}\left(k_{y}^{\prime} \frac{\partial \bar{v}}{\partial y}\right)
\end{aligned}
$$


Since $k_{y}^{\prime} \cong k_{z}^{\prime}$ and $\partial \bar{v} / \partial y \ll \partial \bar{v} / \partial z$, the last term is much smaller than $(\partial / \partial z)\left[k_{z}^{\prime}(\partial \bar{v} / \partial z)\right]$ and can thus be omitted. As a result, we obtain

$$
\begin{aligned}
T_{v}^{\prime}= & \frac{\partial}{\partial z}\left(k_{z} \frac{\partial v^{\prime}}{\partial z}\right)+\frac{\partial}{\partial z}\left(k_{z}^{\prime} \frac{\partial \bar{v}}{\partial z}\right)-\frac{\partial}{\partial z} \overline{\left(k_{z}^{\prime} \frac{\partial v^{\prime}}{\partial z}\right)} \\
& +\frac{\partial}{\partial x}\left(k_{x} \frac{\partial v^{\prime}}{\partial x}\right) .
\end{aligned}
$$

The small-scale turbulent diffusion coefficients in $x$ and $z$ directions are assumed to be equal $\left(k_{x}=k_{z}=k\right)$ above the stratification-dependent surface layer (described below) and are calculated as follows:

$$
k= \begin{cases}\tilde{l}^{2} D^{1 / 2}, & \text { if } D>0 \\ k_{\min }, & \text { if } D \leq 0,\end{cases}
$$

in which $D=2(\partial u / \partial x)^{2}+2(\partial w / \partial z)^{2}+(\partial u / \partial z+\partial w /$ $\partial x)^{2}+(\partial v / \partial x)^{2}+(\partial v / \partial z)^{2}-\operatorname{Pr}(g / \bar{\theta})(\partial \theta / \partial z)$, and $k_{\min }=$ $1 \mathrm{~m}^{2} \mathrm{~s}^{-1}$, where $\operatorname{Pr}$ is the turbulent Prandtl number; $\tilde{l}$ is the mixing length calculated as $\tilde{l}=\chi z /\left(1+\chi z / \tilde{l}_{\infty}\right)$ (Blackadar 1962), where $\chi=0.4$ is von Kármán's constant; and $\tilde{l}_{\infty}$ is the value of the mixing length at the upper boundary of the surface layer and is calculated by matching the values of small-scale turbulent diffusion coefficients calculated using the surface layer model to those calculated using Eq. (17) at the upper boundary of the surface layer.

The stratification-dependent surface layer is described by the Monin-Obukhov similarity theory in which the vertical profiles of velocity, virtual potential temperature $\theta_{v}$, and mixing ratio $q$ are written as

$$
\begin{array}{r}
\frac{\nu}{u_{*}}=\frac{1}{\chi}\left[\ln \left(\frac{z}{z_{0}}\right)+\varphi_{V}\right], \\
\frac{\left(\theta_{v}-\theta_{\mathrm{vs}}\right)}{\theta_{*}}=\frac{R}{\chi}\left[\ln \left(\frac{z}{z_{T}}\right)+\varphi_{\theta}\right], \\
\frac{\left(q-q_{s}\right)}{q_{*}}=\frac{R}{\chi}\left[\ln \left(\frac{z}{z_{q}}\right)+\varphi_{q}\right],
\end{array}
$$

where $\theta_{v s}$ and $q_{s}$ are the corresponding values at the sea surface (strictly speaking, at the levels of $z_{T}$ and $z_{q}$ ); $u_{*}$ is friction velocity; $\theta_{*}$ and $q_{*}$ are the characteristic scales of fluctuations of potential temperature and mixing ratio, respectively; $R$ is constant equal to $0.74 ; z_{0}$ is roughness parameter denoting the level where the velocity vanishes, and $\varphi_{v}, \varphi_{\theta}$, and $\varphi_{q}$ are the universal functions of $\zeta=z / L_{v}$, where $L_{v}=\theta_{\mathrm{vs}} u_{*}^{2} / \chi g \theta_{* v}$ is the Monin-Obukhov length scale (with virtual correction). The universal functions are stratification dependent and are chosen following Businger et al. (1971) and Deardorff (1972); $z_{0}$ is determined using the Charnock (1955) formula. The ratio $z_{0} / z_{T}\left(=z_{0} / z_{q}\right)$ is represented by the universal functions of Prandtl and Reynolds (Re $=z_{0} u_{*} / v$ ) numbers as in Garratt and Hicks (1973) and
Hicks (1975). Three ranges of Re, corresponding to laminar $(\operatorname{Re}<0.1)$, transition $(0.1<\operatorname{Re}<16.3)$, and turbulent regimes $(\operatorname{Re}>16.3)$, are considered. The universal functions within these ranges are chosen based on the results of laboratory experiments and observed data analyzed by Kazakov and Lykosov (1980). In particular, for high Re, typical for strong winds, the following function is used:

$$
\ln \left(z_{0} / z_{T}\right)=a(\operatorname{Re})^{n} \operatorname{Pr}^{m},
$$

where $a=(0.14) 30^{n}, n=0.45, m=0.8$, and $\operatorname{Pr}=$ 0.71 .

The values of $u_{*}, \theta_{* v}$, and $q_{*}$, which determine the surface stress, sensible, and latent heat fluxes correspondingly, are calculated using an iterative procedure with respect to $L_{v}$. Using the value of $L_{v}$ calculated at the previous iteration, new values of $z_{0}, u_{*}, \theta_{* v}$, and $q_{*}$ are calculated using (18)-(19). Knowing these values, a new value of $L_{v}$ is calculated. The iterations continue until the new value of $L_{v}$ becomes close enough to the value obtained at the previous iteration.

Parameterization of CWC is relatively simple and in some respect similar to the large-scale precipitation schemes typically used in general circulation models. When mixing ratio exceeds the saturation value, some fraction of water vapor condenses and temperature increases such that the final state corresponds to $100 \%$ relative humidity. Since small cumuli do not usually precipitate, CWC generated as a result of condensation is simply advected by the wind with no sedimentation. Therefore, the equation for $l$ is similar to that for mixing ratio. Although parameterization of CWC is simple, it takes into account the main thermodynamic effects: heating by condensation of water vapor and cooling by evaporation of CWC.

In summary, the present BL model is represented by two coupled 2D systems of equations describing the evolution of the background flow in the $y-z$ plane and convective motions (large eddies) in the $x-z$ plane. The most important feature of the model is that the terms responsible for the interaction of the background flow and large eddies are calculated explicitly during the integration of these two systems of equations.

\section{b. Computational domains and methods of calculations}

Calculations are conducted on four finite-difference grids: one grid in the $y-z$ plane for the background flow and three grids in the $x-z$ plane for the convective motions placed perpendicular to the $y$ axis at $y=100 \mathrm{~km}$, $y=250 \mathrm{~km}, y=400 \mathrm{~km}$, correspondingly (Fig. 3). The background flow grid consists of 11 grid points along the $y$ axis with a uniform grid spacing of $50 \mathrm{~km}$. In the vertical, it has 61 levels with a uniform 50-m resolution. Each convective grid consists of 257 grid points in the $x$ direction with a resolution of $65 \mathrm{~m}$ and the same vertical levels as the background flow grid. 
Obviously, the high spatial resolution of the convective grids allows for explicit simulations of large eddies in the BL. Some preliminary sensitivity experiments were performed with a smaller size of the convective grid in the $x$ direction $(8.32 \mathrm{~km}$ instead of $16.64 \mathrm{~km})$. The differences in the magnitudes of surface fluxes, as well as second moments were about 15\%-20\%. While these differences are relatively small, the larger, $16.64-\mathrm{km}$, computational area is used in the simulations discussed below.

The governing equations are discretized using the upstream finite differences and integrated in time using the Matsuno scheme (Haltiner and Williams 1980). The nonlinear terms in Eqs. (7), (8), and (10) are discretized using the Arakawa (1966) method which conserves energy and vorticity. The Poisson equation [(9)] is solved using the fast Fourier transform method. The background flow equations and the convective equations are integrated using 5- and 1-s time steps, respectively.

\section{c. Boundary and initial conditions}

\section{1) THE BACKGROUND FLOW EQUATIONS}

At $z=H=3 \mathrm{~km}, \partial \bar{v} / \partial z=0$ is assumed, and $\bar{p}(y$, $H), \bar{\theta}(y, H)$, and $\bar{q}(y, H)$ are prescribed. The gradient of $\bar{p}(y, H)$ varies in different experiments from 0.5 to $3 \mathrm{mb}(100 \mathrm{~km})^{-1} ; \bar{\theta}(y, H)$ is set equal to $312 \mathrm{~K}$, and $\bar{q}(y, H)$ varies in different experiments as discussed below.

At $z=0, \bar{w}=0$, other variables are calculated by the surface layer algorithm described above. The SST varies linearly from $298.8 \mathrm{~K}$ at $y=0$ to $302.8 \mathrm{~K}$ at $y$ $=500 \mathrm{~km}$.

At $y=0, \bar{v}(0, z), \bar{\theta}(0, z)$, and $\bar{q}(0, z)$ are prescribed. Velocity profiles $\bar{v}(0, z)$ vary in different experiments as described below. At $y=500 \mathrm{~km}$ all variables are calculated using the governing equations with no turbulent terms included.

At $t=0, \bar{v}(y, z)$ is obtained from Eq. (5), neglecting the time tendency, convective, and turbulent fluxes. Pressure and vertical velocity are calculated using the static and continuity equations, correspondingly, and $\bar{\theta}(y, z)$ increases linearly with height, representing a stable $\mathrm{BL}$, with $\bar{\theta}(y, H)=312 \mathrm{~K}$ and the initial sea surface-air temperature difference set to $3 \mathrm{~K}$. In the control experiment, relative humidity is equal to $90 \%$ in the lower $500 \mathrm{~m}$ and then decreases linearly to $40 \%$ at $z=H$.

\section{2) The CONVECTIVE EQUATIONS}

At the lateral boundaries, periodic conditions are imposed. At $z=H, v^{\prime}, w^{\prime}, \theta^{\prime}, q^{\prime}, l^{\prime}, \eta^{\prime}$ are set to be equal to 0 . At $z=0, w^{\prime}=0$. The sea surface turbulent fluxes are determined using the surface layer algorithm described above. At $t=0$, the convective motions are triggered by a sinusoidal humidity impulse with an amplitude of $0.1 \mathrm{~g} \mathrm{~kg}^{-1}$ at $z=100 \mathrm{~m}$.

\section{Design of numerical experiments}

The numerical experiments are designed to simulate the BL conditions resembling those in tropical cyclones. The effects of different initial velocity profiles, air subsidence (mean vertical velocities), and latent heat release (via variation of the air humidity) are investigated. The sequence of calculations is as follows. The background flow equations are integrated first. At this stage, the terms representing the effects of convective motions on the background flow, that is, $\partial \overline{\left(w^{\prime} v^{\prime}\right)} / \partial z \partial \overline{\left(w^{\prime} \theta^{\prime}\right)} / \partial z$, and $\partial \overline{\left(w^{\prime} q^{\prime}\right)} / \partial z$, etc., are set equal to 0 , and the only subgrid process considered is small-scale turbulence. After the background flow reaches a quasi-steady state (typically in less than $4 \mathrm{~h}$ ), the convective equations are switched on at $t=4 \mathrm{~h}$, and the two systems of equations are solved simultaneously. During the period of a 5-s time step of the integration of the background flow equations, the convective equations are integrated using a 1-s time step. Convective fluxes $\partial \overline{\left(w^{\prime} v^{\prime}\right)} / \partial z, \partial \overline{\left(w^{\prime} \theta^{\prime}\right)} / \partial z$, and $\partial \overline{\left(w^{\prime} q^{\prime}\right)} / \partial z$ are calculated for each of the three convective grids using the averaging (1) and assuming that the width $L$ is equal to the size of the convective domain in the $x$ direction. These fluxes are assigned to the corresponding locations of the convective grids $(y=100$ $\mathrm{km}, y=250 \mathrm{~km}, y=400 \mathrm{~km}$ ) and then interpolated using cubic splines along the $y$ direction onto the grid points of the background flow. They are subsequently used in the ensuing time step of the background flow equations. The newly calculated background flow values are used for succeeding integration of the convective equations.

\section{Results}

We now discuss the results of a series of numerical experiments conducted to investigate possible mechanisms responsible for formation of large eddies in the marine boundary layer, resembling tropical cyclone conditions. Our primary focus is the effects of large eddies on the wind, temperature, and humidity profiles of the background flow and air-sea fluxes. We will also discuss the sensitivity to various background flow characteristics such as the initial wind profiles, background flow vertical velocity, and humidity.

\section{a. Control experiments}

Initial background velocity profiles corresponding to weak (maximum of $20 \mathrm{~m} \mathrm{~s}^{-1}$ ), moderate (maximum of $40 \mathrm{~m} \mathrm{~s}^{-1}$ ), and strong (maximum of $65 \mathrm{~m} \mathrm{~s}^{-1}$ ) wind speeds are investigated. The initial profiles, shown in Figs. $4 \mathrm{a}-\mathrm{c}$ by dotted lines, have a very simple form. In the week wind case, the speed linearly increases from $z=0 \mathrm{~km}$ to $z=3 \mathrm{~km}$, and the vertical wind shear is set to be very small. In the cases of intermediate and strong wind, the wind speed linearly increases from $z$ $=0 \mathrm{~km}$ to $z=2 \mathrm{~km}$ and then linearly decreases from 

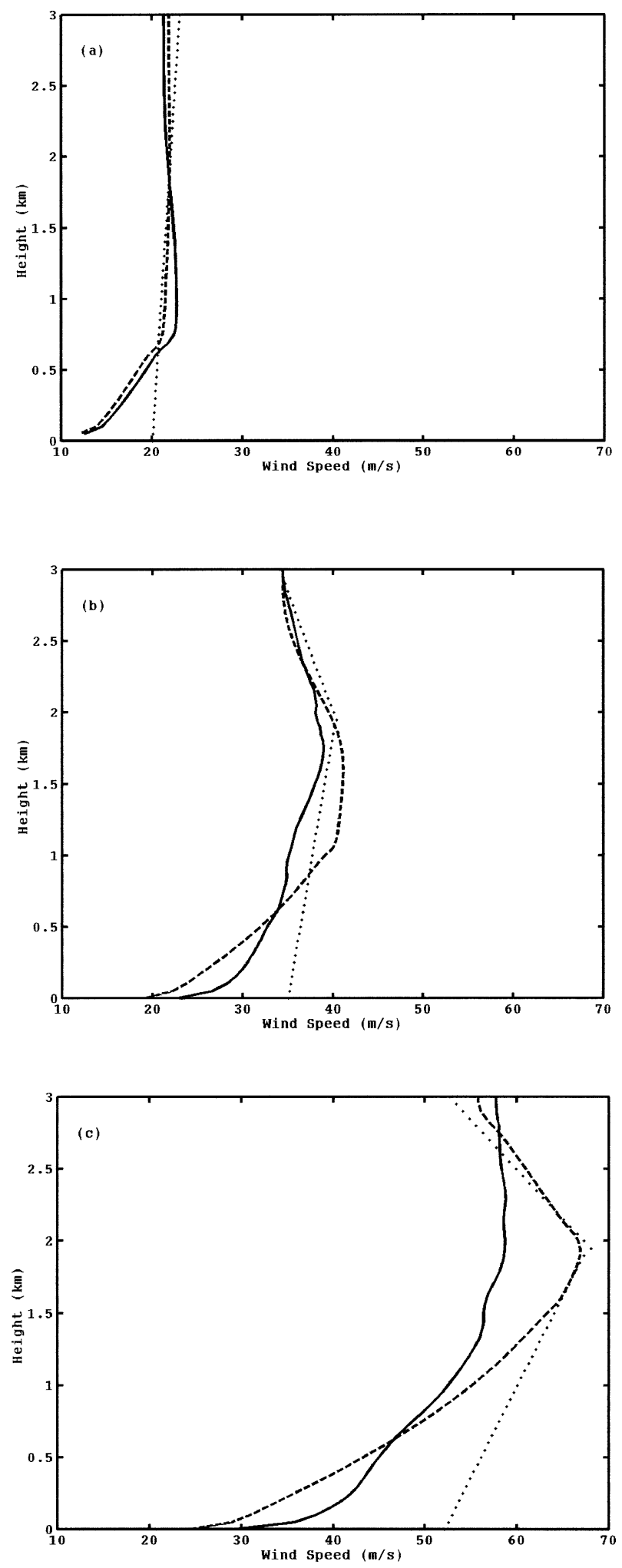

FIG. 4. Vertical profiles of the background velocity $\bar{v}(y, z)$ at $y=$ $400 \mathrm{~km}$ for (a) weak wind, (b) moderate wind, and (c) strong wind. Dotted curves are initial profile, dashed curves are stationary state $(t$ $=4 \mathrm{~h}$ ), and solid curves are $4 \mathrm{~h}$ after convection was switched on $(t=8 \mathrm{~h})$.

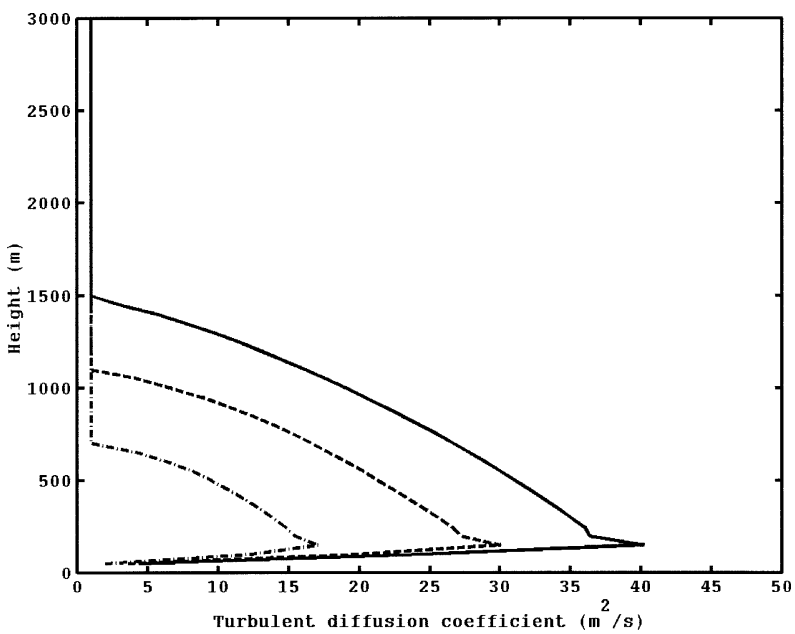

FIG. 5. Vertical profiles of the turbulent diffusion coefficient in the weak (dashed-dotted line), moderate (dashed line), and strong wind (solid line) cases at $t=4 \mathrm{~h}$ (no convection).

$z=2 \mathrm{~km}$ to $z=3 \mathrm{~km}$. The height of the wind speed maximum of $2 \mathrm{~km}$ roughly corresponds to that observed at the periphery of tropical cyclones (Kepert and Wang 2001).

Figures $4 \mathrm{a}-\mathrm{c}$ show $\bar{v}(z)$ at $t=4 \mathrm{~h}$, shortly after the background flow reached a quasi-stationary state, and at $t=8 \mathrm{~h}, 4 \mathrm{~h}$ after the convective equations are switched on. During the first 4-h period, the velocity profiles are mostly modified in the lower part of the BL, showing about a 50\% decrease of the near-surface wind speed. This is due to surface friction and small-scale vertical turbulent mixing. As a result, vertical velocity shears develop in the lower part of the BL, with the strongest shears occurring for the higher initial wind speeds. After the convective equations are switched on, the vertical velocity profiles are further altered because of the development of large eddies in the BL, especially noticeable in the moderate and strong wind cases. In those cases, the wind speeds near the surface are increased by about 5 and $10 \mathrm{~m} \mathrm{~s}^{-1}$, respectively, accompanied by a decrease of the wind speed maximum above. In the moderate wind case, the background velocity profiles are modified up to about $2 \mathrm{~km}$, while in the strong wind case the large eddies' effect extends to the entire BL. It is evident that the large eddies play a very small role in the weak wind case.

Figures 5 and 6 illustrate the primary mechanisms through which large eddies are generated in our simulations. Previous studies indicate that rolls can be generated by either dynamic instability when velocity changes with height in such a way that an inflection point occurs in the velocity component normal to the roll axis or by thermal, Rayleigh-Bernard instability when the BL is heated from below and cooled from above (Etling and Brown 1993). Figure 5 shows the vertical profiles of the small-scale turbulent diffusion coefficient at $t=4 \mathrm{~h}$. While in the weak wind case, $k$ 


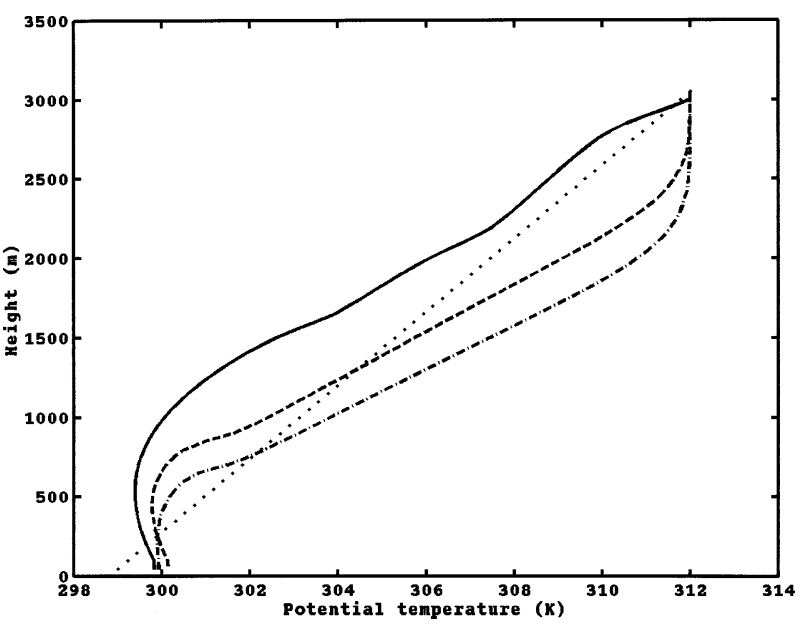

FIG. 6. Potential temperature profiles for the weak (dashed-dotted), moderate (dashed), and strong (solid) wind conditions at $t=4 \mathrm{~h}$. The initial potential temperature profile is shown by the dotted line.

does not exceed $15 \mathrm{~m}^{2} \mathrm{~s}^{-1}$, in the strong wind case, the maximum of $k$ reaches $40 \mathrm{~m}^{2} \mathrm{~s}^{-1}$ and remains large up to $1200-1300 \mathrm{~m}$. This intense turbulent mixing is induced by the strong wind shear in the low part of the lower troposphere. Note that $k$ is very small and constant in the upper part of the boundary layer. With height, the gradient of the background velocity $\partial \bar{v} / \partial z$ decreases, while the gradient of the potential temperature $\partial \bar{\theta} / \partial z$ [as well as the stability parameter $\operatorname{Pr}(g / \bar{\theta})(\partial \theta / \partial z)$ in (17)] remains fairly uniformed. Above a certain height, parameter $D$ in (17) becomes less than zero and $k$ becomes equal to its minimum value $k_{\min }=\mathrm{m}^{2} \mathrm{~s}^{-1}$.

As a result of the strong turbulent mixing, a mixed layer with virtually constant potential temperature is formed (Fig. 6) by $t=4 \mathrm{~h}$. The mixed-layer depth increases with an increase of the background wind. The reduced stability and the strong velocity shear create favorable conditions for dynamic instability that trigger the formation of convective motions in the BL when the convective equations are switched on Figure 7 shows time dependence of the mean kinetic energy of the convective motions calculated as $\mathrm{KE}=1 / 2 N \Sigma_{i}\left(u_{i}^{\prime 2}+\right.$ $v_{i}^{\prime 2}+w_{i}^{\prime 2}$ ) (where $N$ is the total number of grid points) in the three analyzed cases. The kinetic energy is the largest in the strong wind case with the mean value of the velocity perturbations reaching $3-4 \mathrm{~m} \mathrm{~s}^{-1}$.

Besides the buoyancy appearing in the dynamically induced mixed layer, there is an additional source of the kinetic energy of convective motions in the present model. In the moderate and strong wind cases, the top of the mixed layer exceeds the cloud-base level ( 0.9 $\mathrm{km})$, which leads to formation of clouds. The associated latent heat release provides remarkable source of energy to large eddies. In the weak wind case, the mixed-layer depth is well below the cloud base. As a result, no clouds and therefore no latent heat release arise in this case.

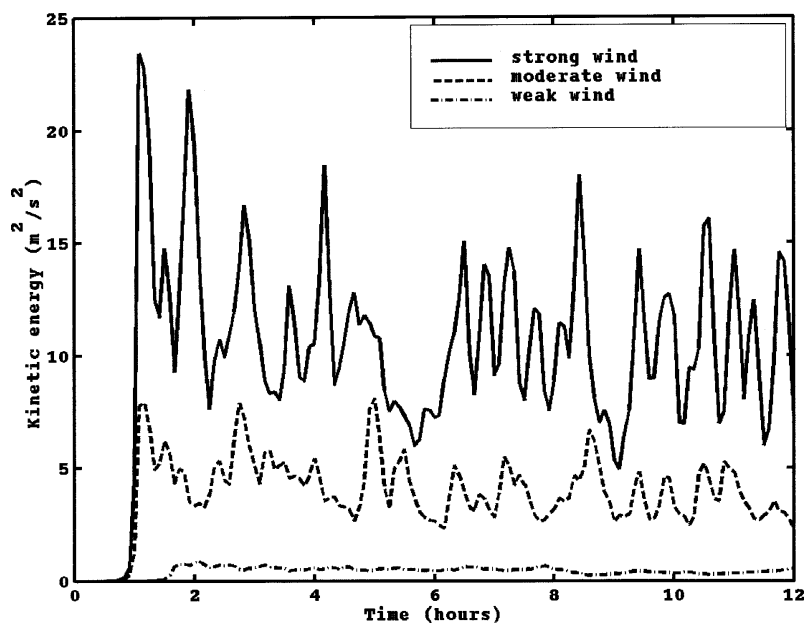

FIG. 7. Time dependence of the kinetic energy of convective motions in the weak (dashed-dotted), moderate (dashed), and strong (solid) wind cases. Time $t=0$ corresponds to the beginning of the integration of the convective equations.

We will discuss the role of air humidity in the large eddy formation in greater detail below.

We now consider the contribution of different terms to the changes of $\bar{v}(z)$ in Eq. (5) when the background flow equations are integrated simultaneously with the convective equations. Figure 8 shows the time integrated $-(1 / \rho)(\partial \bar{P} / \partial y), \bar{T}_{v}$, and $-\left[\partial \overline{\left(w^{\prime} v^{\prime}\right)}\right] / \partial z$ during the period from $t=4 \mathrm{~h}$ to $t=6 \mathrm{~h}$. We can immediately see that the convective momentum flux is negligible in the weak wind case but it dominates other terms in the strong and moderate wind conditions. From reviewing the convective momentum influx vertical profiles, it is apparent that it is primarily responsible for the modification of the background velocity profiles seen in Fig. 4 , that is, an increase (decrease) of $\bar{v}(z)$ in the lower (upper) part of the BL.

Contribution of the time-integrated temperature and humidity convective influxes $\int_{t=4 \mathrm{~h}}^{t=6 \mathrm{~h}} \partial \overline{\left(w^{\prime} \theta^{\prime}\right)} / \partial z d t$ and $\int_{t=4 \mathrm{~h}}^{t=6 \mathrm{~h}}$ $\partial \overline{\left(w^{\prime} q^{\prime}\right)} / \partial z d t$ to the changes of $\theta$ in Eq. (6) and $\bar{q}$ in a similar equation for mixing ratio are presented in Fig. 9. While the contribution of these fluxes is negligible in the weak wind case, they are mainly responsible for the vertical transport of heat and humidity in the BL in the moderate and strong wind conditions. This is clearly seen in Fig. 10, where the vertical profiles of $\bar{\theta}(z)$ and $\bar{q}(z)$ are shown at $t=4 \mathrm{~h}$ and $t=6 \mathrm{~h}$. In the strong wind case, one can see an increase (decrease) of potential temperature and a decrease (increase) of mixing ratio in the lower (upper) part of the BL in correspondence to the vertical profiles of the convective fluxes in Fig. 9.

One of the most important effects of the large eddies found in these simulations is a significant increase of the latent heat flux at the sea surface. Figures 11a and $11 \mathrm{~b}$ show time dependence of the sensible and latent heat fluxes after the convective equations are switched on. It is seen that the large eddies are responsible for 

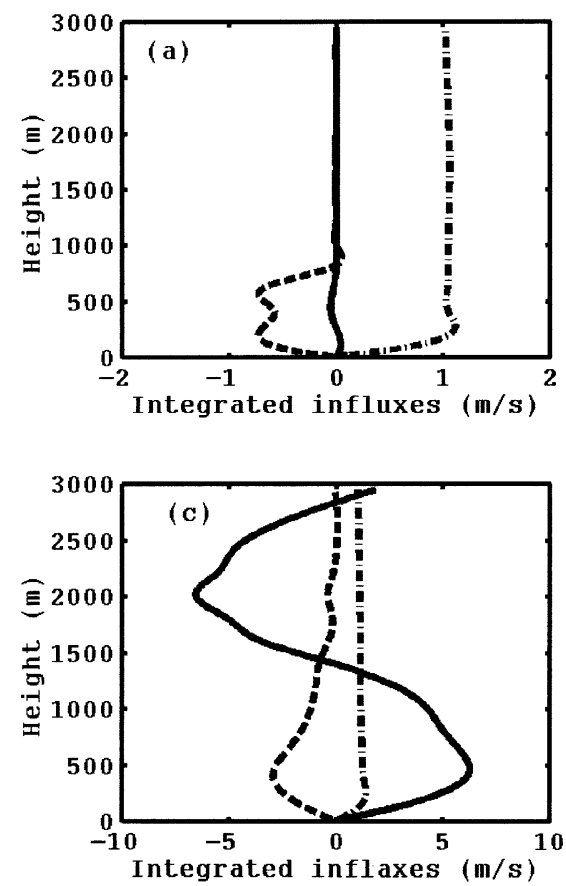

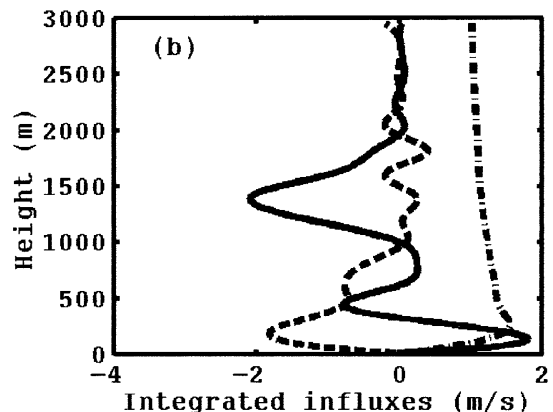

FIG. 8. Contribution of different factors to the changes of the background velocity for $2 \mathrm{~h}$ after the convective equations were switched on for (a) weak, (b) moderate, and (c) strong wind. The factors are pressure gradient $-\int_{t=4 \mathrm{~h}}^{t=6 \mathrm{~h}} \partial \bar{P} / \partial y d t$ (dashed-dotted line), small-scale turbulence flux $\int_{t=4 \mathrm{~h}}^{t=6 \mathrm{~h}} \bar{T}_{v} d t$ (dashed line), and convective-scale momentum flux $-\int_{t=4 \mathrm{~h}}^{t=6 \mathrm{~h}} \partial \overline{\left(w^{\prime} v^{\prime}\right)} / \partial y d t$ (solid line).
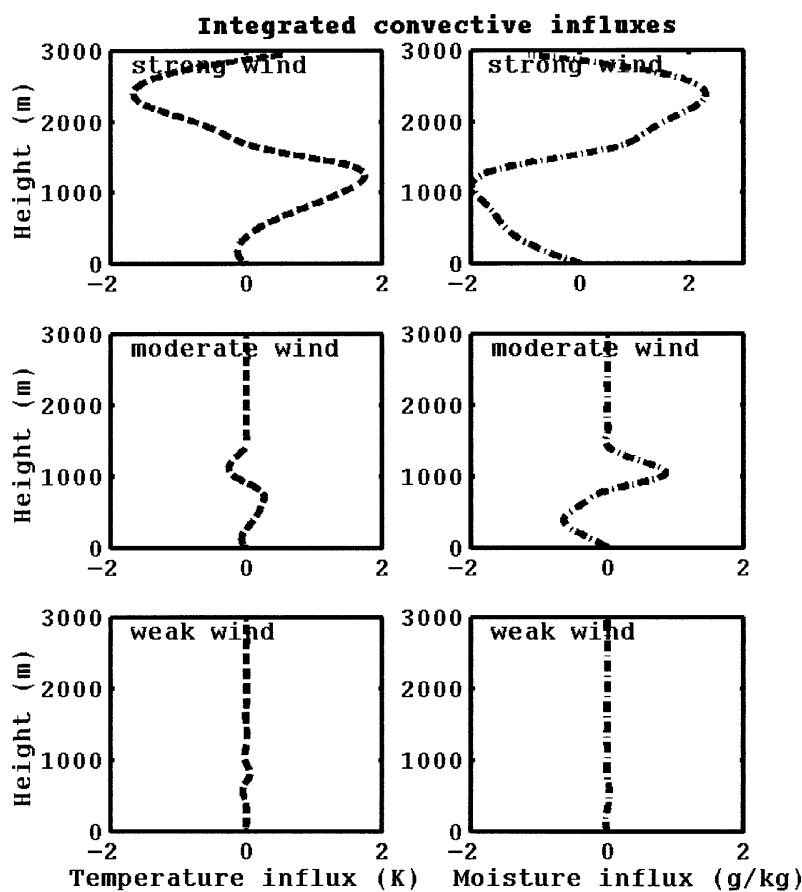

FIG. 9. Contribution of the (left) convective temperature influx

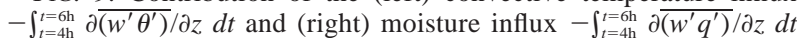
to the changes of the background potential temperature and mixing ratio, correspondingly, for strong, moderate, and weak wind cases. The integration time is $2 \mathrm{~h}$ after the convective equations were switched on.
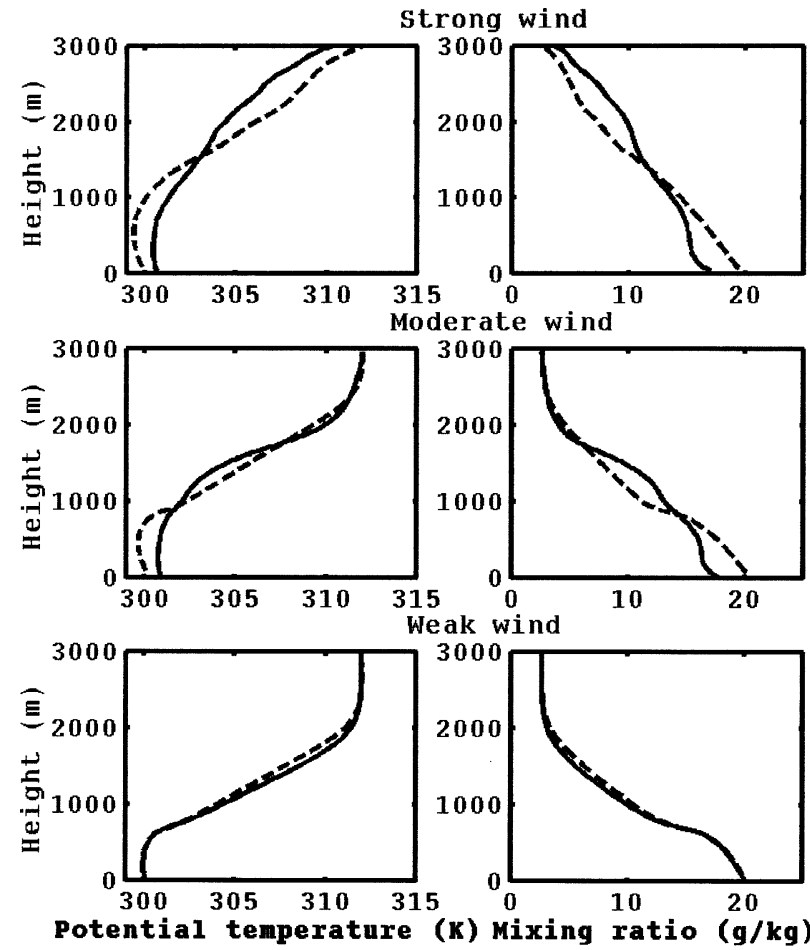

FIG. 10. Vertical profiles of the (left) background potential temperature and (right) mixing ratio for the strong, moderate, and weak wind cases. Dashed curves denote the profiles at $t=4 \mathrm{~h}$ (no convection) and solid curves show the profiles at $2 \mathrm{~h}$ after the convection was switched on $(t=6 \mathrm{~h})$. 

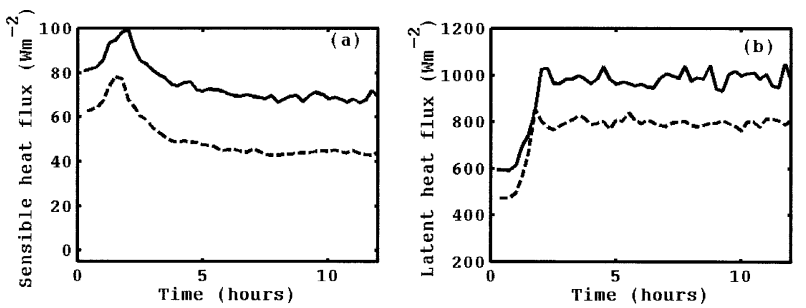

FIG. 11. Time dependence of the (a) sensible and (b) latent heat fluxes at the sea surface after the convective equations are switched on for the moderate (dashed line) and strong (solid line) wind cases. The horizontal axis indicates the elapsed time from the beginning of the integration of the convective equations.

almost doubling of the latent heat flux. Such a strong effect can be attributed to an increase in the difference between saturated mixing ratio at the sea surface and mixing ratio of air in the surface layer. Contrarily, the sensible heat flux, which is much smaller than the latent heat flux, is decreased somewhat due to an increased air temperature in the surface layer and hence reduced air-sea temperature difference.

Figure 12 shows the streamfunction, vertical velocity, and $\mathrm{CWC}$ in the third convective grid in the strong wind case at $t=8 \mathrm{~h}$. One can see that $4 \mathrm{~h}$ after the convective equations are switched on two distinct roll vortices have developed. They are separated by about $5 \mathrm{~km}$ and extended to about $2.7 \mathrm{~km}$ in height. The vertical velocities in the rolls reach about $3-4 \mathrm{~m} \mathrm{~s}^{-1}$ and the maximum values of CWC (equal in our case to the maximum of liquid water content) in cumulus clouds reach about 1.5$2 \mathrm{~g} \mathrm{~kg}^{-1}$. There are numerous observational and numerical studies in which the value of ratio $-z_{i} / L_{v}$ (where $z_{i}$ is the BL depth, or inversion level) has been used as a criterion for the formation of roll vortices. For example, using a 3D model, Deardorff (1972) obtained the roll structure at $-z_{i} / L_{v}<25$. LeMone (1973) found in an observational study that rolls were formed in nearly neutral conditions at $-z_{i} / L_{v}<10$. In our simulations, the values of $-z_{i} / L_{v}$ did not exceed 4 (if $z_{i}$ is taken equal to $3 \mathrm{~km}$ ) in the moderate and strong wind cases. If $z_{i}$ is determined by the inversion (or stable layer) base the values $-z_{i} / L_{v}$ do not exceed $\sim 3$, which is consistent with other studies that simulated the formation of roll vortices (Moeng and Sullivan 1994; Glendening 2000).

\section{b. Sensitivity experiments}

In the control experiments the convective equations are switched on after the background flow achieves a quasi-steady state at $t=4 \mathrm{~h}$. This is done to isolate the effects of large eddies on the BL structure. Since in reality the interaction between different scales in the BL takes place all the time, it is interesting to compare the results of the control experiments with those when the convective equations are switched on at $t=0$. Figures $13 \mathrm{a}-\mathrm{c}$ compare the vertical profiles of background velocity, potential temperature, and mixing ratio obtained
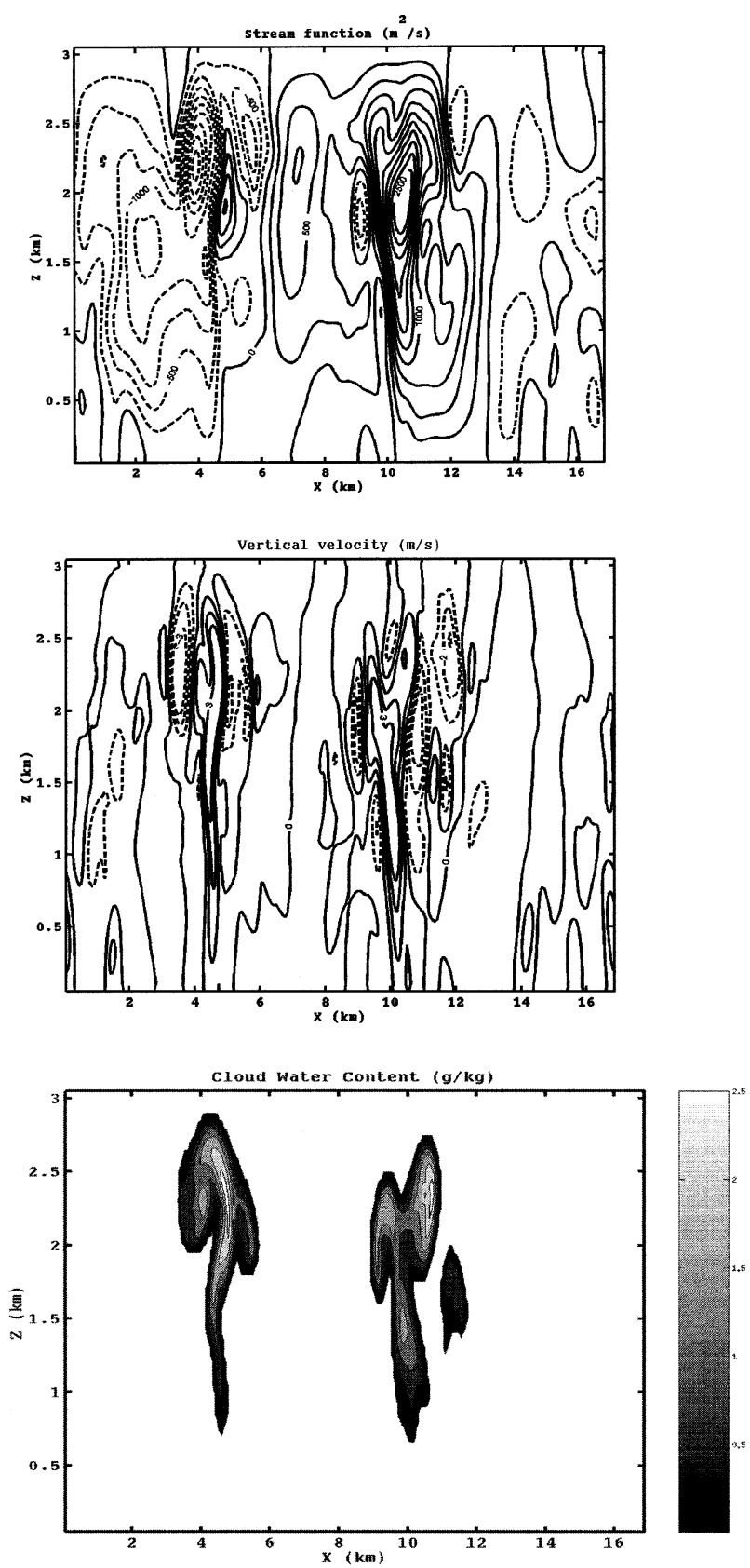

FIG. 12. (top) Streamfunction, (middle) vertical velocity, and (bottom) cloud water content in the third convective grid for the strong wind case at $t=8 \mathrm{~h}$. Solid lines indicate positive values; dashed lines indicate negative values.

at $t=8 \mathrm{~h}$ for the strong wind speed conditions when the convective equations are switched on at $t=4 \mathrm{~h}$ (dashed line) and at $t=0$ (solid line). After some transient period the results of both simulations converge, leading to similar vertical profiles. We can therefore conclude that the main effects of large eddies on the structure of the BL are not very sensitive to the time when the convective motions are initiated.

We now investigate the sensitivity of the formation 
(a)

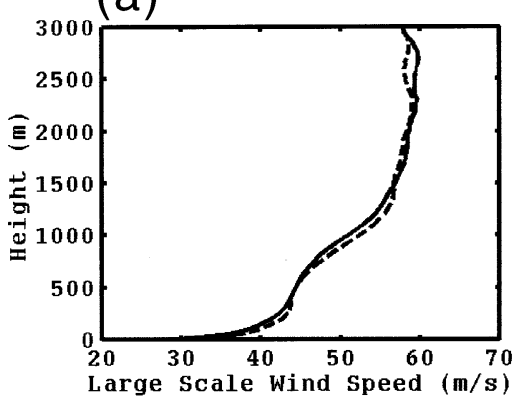

(c)

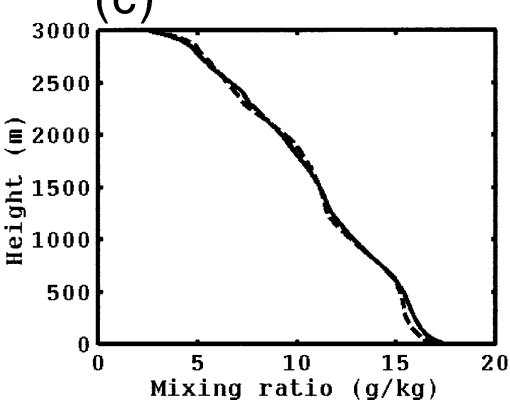

(b)

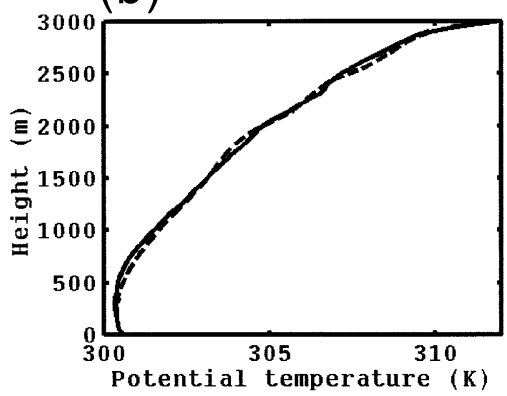

control experiment --- C+LS experiment

FIG. 13. Vertical profiles of the (a) background velocity, (b) potential temperature, and (c) mixing ratio at $t=8 \mathrm{~h}$ in the strong wind case when the convective equations are switched on at $t=4 \mathrm{~h}$ (solid line) and $t=0$ (dashed line).

of large eddies to the intensity of subsidence in the BL. Subsidence in the model is controlled by the pressure gradient along the $y$ axis at the upper boundary of the computational area, $z=H$. For example, an increase of the pressure gradient will result in an acceleration of the background flow, leading to an increase in flow divergence in the BL and a corresponding increase of the subsidence velocity. In the strong wind case the mean subsidence velocity at $y=400 \mathrm{~km}$ reaches about 1.5 $\mathrm{cm} \mathrm{s}^{-1}$. In this sensitivity experiment, we increase the pressure gradient at $z=3 \mathrm{~km}$ from 0.5 to $3 \mathrm{mb}(100$ $\mathrm{km})^{-1}$. As a result, the mean subsidence velocity is increased to $15 \mathrm{~cm} \mathrm{~s}^{-1}$ (not shown). The velocity profile at the boundary $y=0, \bar{v}(0, z)$, is also specified in such a way that at the end of the background flow integration the velocity profile at $y=400 \mathrm{~km}, \bar{v}(400, z)$, becomes similar to that in the control run. This is done to assure that the differences in the convective motions arising in the third convective grid are primarily due to the increased air subsidence. Figures $14 \mathrm{a}-\mathrm{c}$ show the streamfunction, vertical velocity, and $\mathrm{CWC}$ in the third convective grid at $t=8 \mathrm{~h}$. Although subsidence is an order of magnitude stronger than in the control case, large eddies are still formed under the strong wind conditions. However, they are significantly weaker and mostly confined to the lower part of the computational area, up to $1.7 \mathrm{~km}$. As in the control case, the near-surface wind speed is increased due to the large eddies but it is smaller, about $7 \mathrm{~m} \mathrm{~s}^{-1}$ (not shown).

Another sensitivity experiment is conducted to investigate the role of latent heat release (through varia- tion of air humidity) in the formation of large eddies in strong wind conditions. There are several sources of humidity in our model: humidity specified at $t=0$, evaporation from the sea surface and advection of humidity through the boundary condition at $y=0$. Supplemental runs (not shown) indicate that if we only reduce humidity at $t=0$ the development of large eddies is delayed by a few hours but their final intensity, measured by kinetic energy of the convective motions, is nearly the same to the control experiments. This indicates that the main sources of humidity are the sea surface evaporation and horizontal advection. Therefore, in order to investigate the effect of reduced humidity in this experiment we (a) switch off evaporation from the sea surface and (b) reduce relative humidity at the boundary $y=0$ to $40 \%$ at $z=0$ and to $30 \%$ at $z=3$ $\mathrm{km}$. Figure 15 shows the time dependence of kinetic energy of the convective motions in this experiment and the control case. It is evident that despite the strong wind shear, the formation of large eddies is substantially hampered by humidity reduction in the BL. Because of reduced humidity the cloud-base level is increased and becomes larger than the height of the mixed layer. As a result, clouds do not form and thus the energy source provided by latent heat release is virtually eliminated.

Note that we simulate here the structure of the BL limited from above by an inversion or by a stable layer. This may correspond to the region outside of the hurricane eyewall where the boundary layer inversion hardly exists. However, the zone of strong updrafts in the eyewall is comparatively narrow. Therefore, our sim- 

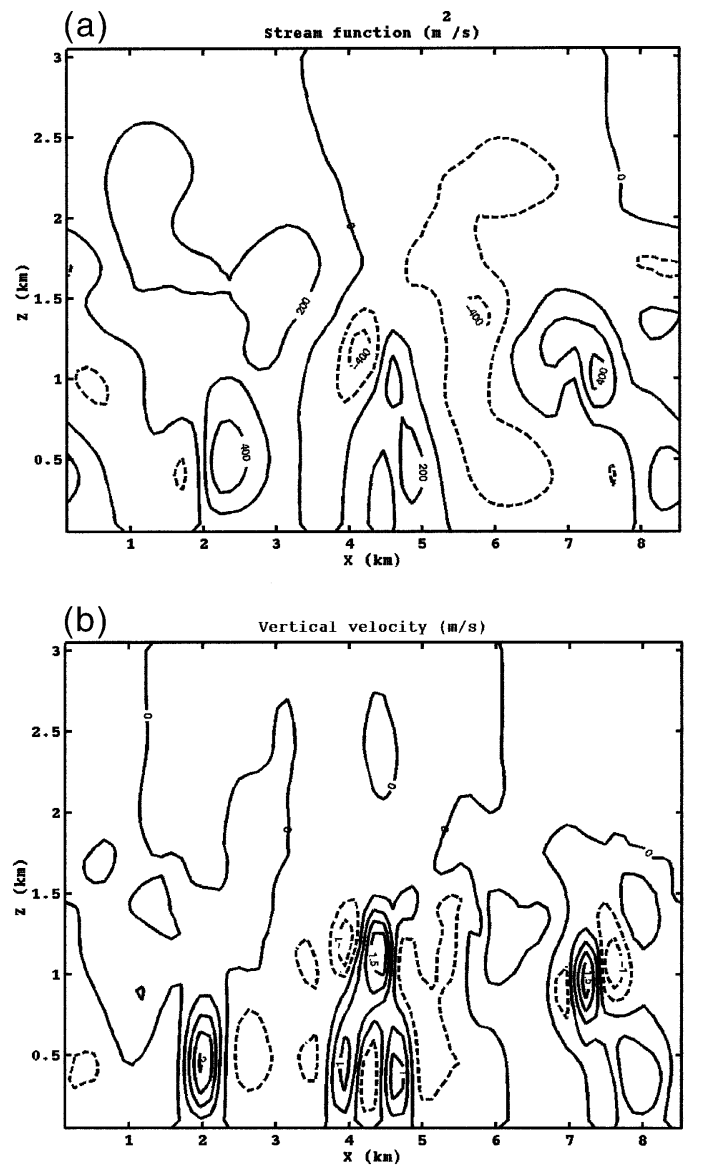

(c)

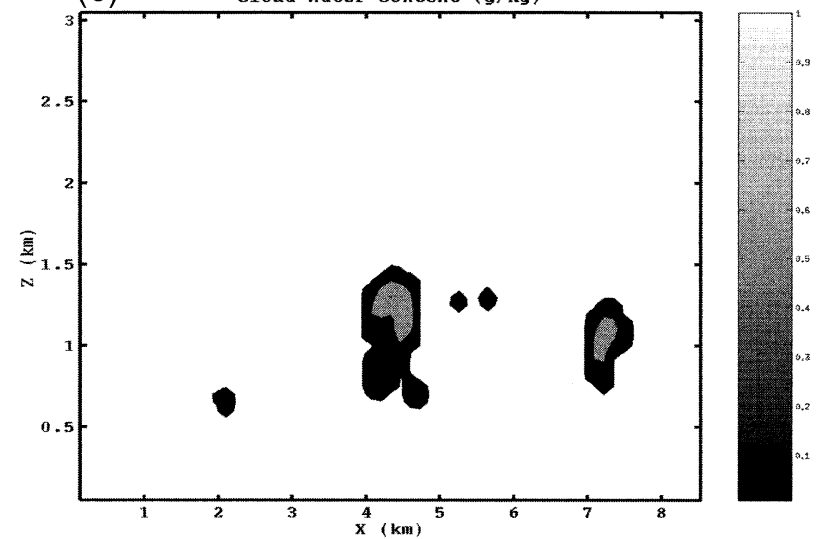

FIG. 14. (a) Streamfunction, (b) vertical velocity, and (c) cloud water content for the case of increased subsidence at $t=8 \mathrm{~h}$. Solid lines indicate positive values; dashed lines indicate negative values.

ulations can be applied even within quite small distances from the hurricane center. In the vicinity of the hurricane core region the wind speed maximum is typically located in the lower part of the BL, near the height of 500-700 $\mathrm{m}$ (Powell et al. 2003). To simulate the formation and effects of large eddies under these conditions, an additional experiment is conducted, which is

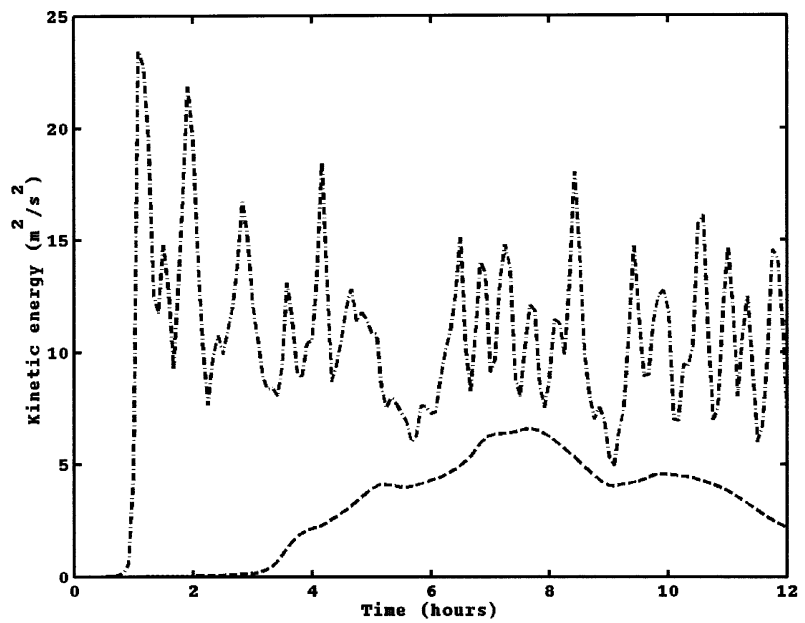

FIG. 15. Time dependence of the kinetic energy of convective motions in the control, strong wind case (dashed-dotted line), and the reduced humidity case (dashed line).

the same as the control, strong wind case except using the initial $\bar{v}(z)$ as shown in Fig. 16 (dotted curve). During the first $4 \mathrm{~h}$ of integration, the height of the wind speed maximum in the background flow increases from 500 to about $1300 \mathrm{~m}$ (Fig. 16, dashed curve). The high winds in the lower part in the BL cannot be supported by the pressure gradient in this model, as it typically occurs in the vicinity of a hurricane eyewall. Nevertheless, one can see that a significant wind shear has developed in the lower part of the BL at $t=4 \mathrm{~h}$. As a result, when the convective equations are switched on, the effect of large eddies on the background velocity profile is even more pronounced than in the control case (Fig. 16, solid curve): the near-surface wind speed increases by more that $12 \mathrm{~m} \mathrm{~s}^{-1}$, while the maximum wind speed decreases by about $10 \mathrm{~m} \mathrm{~s}^{-1}$.

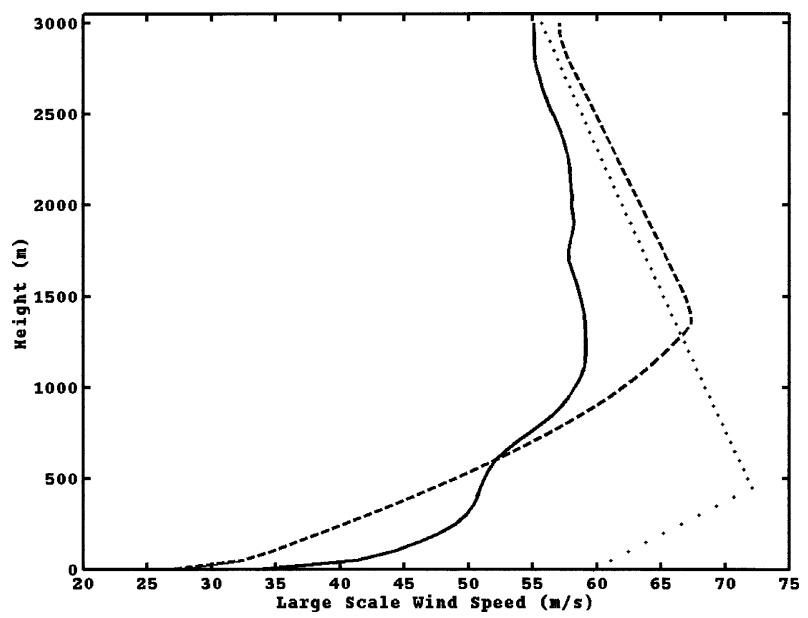

FIG. 16. Vertical profiles of the background velocity $\bar{v}(y, z)$ at $y$ $=400 \mathrm{~km}$ with the initial profile shown by dotted curve. Dashed curve: stationary state $(t=4 \mathrm{~h})$, solid curve: $4 \mathrm{~h}$ after the convection was switched on $(t=8 \mathrm{~h})$. 


\section{c. A remark on the "effective" diffusion coefficient}

We have shown in this study that large eddies can strongly increase vertical mixing and air-sea fluxes, as well as the BL depth in very high wind conditions. It is, therefore, important to include these effects in the future tropical cyclone models to improve the forecast skill. The question arises whether the convective motions can be parameterized within the framework of $K$ theory by replacing the small-scale turbulent coefficient by the "effective" diffusion coefficient. We believe it is hardly possible. There is the fundamental difference in the description of the convective motions and the small-scale turbulence in the BL. The vertical transport of heat, moisture, and momentum due to large eddies is determined by the whole structure of the BL, and therefore cannot be calculated using the local gradients of the mean values. In some situations the convective fluxes can be directed against the gradient of the mean values [see concept of countergradient in Holtslag and Moeng (1991)], which would lead to the negative effective diffusion coefficient. As shown in our experiments, thermodynamic processes, including the formation of clouds and associated latent heat release, play a very important roll in the development of large eddies. These processes are controlled by the whole structure of the BL and air-sea fluxes.

\section{Summary}

We have presented a model of the atmospheric boundary layer that explicitly calculates a two-way interaction of the background flow and convective motions. The model is utilized for investigation of the formation of large eddies (roll vortices) and their effects on the structure of the marine boundary layer under conditions resembling those of tropical cyclones. The model is configured to simulate the initially stably stratified BL that is observed in the regions outside of the eyewall and characterized by air subsidence and decreased SSTs due to the air-sea interaction.

Based on the results of numerical simulations new mechanisms causing the formation of large eddies in the initially stably stratified BL are found. It is shown that two main factors controlling the formation of large eddies are the magnitude of the background wind speed and latent heat release. When the wind speed is high enough, a strong vertical wind shear develops in the lower part of the BL that triggers small-scale turbulent mixing and the formation of a mixed layer. As a result, the vertical profiles of velocity, potential temperature, and mixing ratio in the background flow are modified to allow for the development of large eddies. We can thus conclude that a strong background wind is the necessary condition for the formation of large eddies in the stable stratified BL. The presence of sufficiently large humidity is necessary to provide the major energy source to large eddies through latent heat release in clouds. The depth of the mixed layer increases with an increase in the initial background wind speed. When the top of the mixed layer exceeds the cloud-base level, convective clouds arise and associated latent heat release supplies energy for dramatic intensification of the convective motions.

The numerical experiments show that as soon as large eddies arise, they affect the vertical transport of heat, moisture, and momentum and thus modify the structure of the BL and the air-sea fluxes. The most important manifestation of these effects is a significant increase of the near-surface wind speed, which can exceed $10 \mathrm{~m}$ $\mathrm{s}^{-1}$, and evaporation from the sea surface, which can double for strong winds. These results thus demonstrate an important role that large eddies play in high wind speed conditions. Inclusion of these effects in the BL parameterizations of tropical cyclone models may potentially lead to substantial improvements in the prediction of storm intensity. Note that a significant increase in the near-surface winds due to large eddies begins when the wind speed exceeds $40-50 \mathrm{~m} \mathrm{~s}^{-1}$. This may explain why the operational GFDL/URI hurricane model, which does not consider these effects, has a tendency to underestimate the surface wind speeds for strong hurricanes.

The splitting a 3D system of equations on two coupled subsystems for calculation of the background flow and convective-scale motions suggested in this paper may be applied for the BL parameterization of coherent structures in mesoscale and large-scale atmospheric models. The resolvable $3 \mathrm{D}$ mesoscale velocity field in such models can serve as the background flow. Besides tropical cyclone modeling and forecasting, this approach could also be useful for investigation of interactions of convective and mesoscale processes in other atmospheric phenomena such as extratropical storms, cold-air outbreaks, and sea breezes.

Acknowledgments. The constructive comments received from three anonymous reviewers led to substantial improvements in the presentations of sections 2 and 5. We are grateful for their careful review of the original manuscript. Isaac Ginis was partially supported by the National Science Foundation through Grant ATM 0001038. Alexander Khain and Elena Morozovsky were supported by the Lady Davis Foundation and the U.S.Israel Binational Science Foundation through Grant 2000215.

\section{REFERENCES}

Arakawa, A., 1966: Computational design of long term numerical integration of the equation of fluid motion. I. Two-dimensional incompressible flow. J. Comput. Phys., 1, 119-143.

Atlas, D., B. Walter, C. Shu-Hsieu, and P. J. Sheu, 1986: The structure of the unstable marine boundary layer viewed by lidar and aircraft observations. J. Atmos. Sci., 43, 1301-1318.

Bao, J.-W., J. M. Wilczak, J.-K. Choi, and L. H. Kantha, 2000: Numerical simulations of air-sea interaction under high wind con- 
ditions using a coupled model: A study of hurricane development. Mon. Wea. Rev., 128, 2190-2210.

Bender, M. A., and I. Ginis, 2000: Real-case simulations of hurricaneocean interaction using a high-resolution coupled model: Effects on hurricane intensity. Mon. Wea. Rev., 128, 917-946.

Blackadar, A. K., 1962: The vertical distribution of wind and turbulent exchange in a neutral atmosphere. J. Geophys. Res., 67, 30953102 .

Brown, R. A., 1974: Analytical Methods in Planetary BoundaryLayer Modeling. Adam Hilger, $148 \mathrm{pp}$.

Businger, J. A., J. C. Wyngard, Y. Izumi, and E. F. Bradley, 1971: Flux profile relationship in the atmospheric surface layer. $J$. Atmos. Sci., 28, 181-189.

Charnock, H., 1955: Flux gradient relations near the ground in unstable conditions. Quart. J. Roy. Meteor. Soc., 81, 639-645.

Deardorff, J. W., 1972: Parameterization of the planetary boundary layer for use in general circulation models. Mon. Wea. Rev., 100, 93-106.

Emanuel, K. A., 1994: Atmospheric Convection. Oxford University Press, 580 pp.

_ 1995: Sensitivity of tropical cyclones to surface exchange coefficients and a revised steady-state model incorporating eye dynamics. J. Atmos. Sci., 52, 3969-3976.

Etling, D., and R. A. Brown, 1993: A Review of large-eddy dynamics in the planetary boundary layer. Bound.-Layer Meteor., 65, 215248.

Garratt, J. R., 1992: The Atmospheric Boundary Layer. Cambridge University Press, $316 \mathrm{pp}$.

_ and B. B. Hicks, 1973: Momentum, heat and water vapor transfer to and from natural and artificial surfaces. Quart. J. Roy. Meteor. Soc., 99, 680-687.

Glendening, J. W., 2000: Budgets of lineal and nonlineal turbulent kinetic energy under strong shear conditions. J. Atmos. Sci., 57, 2297-2318.

Grabowski, W. W., 2001: Coupling cloud processes with the largescale dynamics using the Cloud-Resolving Convection Parameterization (CRCP). J. Atmos. Sci., 58, 978-997.

Haltiner, G. J., and R. T. Williams, 1980: Numerical Prediction and Dynamic Meteorology. J. Wiley and Sons, $477 \mathrm{pp}$.

Hicks, B. B., 1975: A procedure for the formulation of bulk transfer coefficients over water. Bound.-Layer Meteor., 8, 515-524.

Holtslag, A. A. M., and C.-H. Moeng, 1991: Eddy diffusivity and countergradient transport in the convective atmospheric boundary layer. J. Atmos. Sci., 48, 1690-1698.

Hong, S.-Y., and H.-L. Pan, 1996: Non-local boundary layer vertical diffusion in a medium-range forecast model. Mon. Wea. Rev., 124, 2322-2339.

Katsaros, K. B., P. Vachon, P. G. Black, P. P. Dodge, and E. W. Uhlhorn, 2000: Wind fields from SAR: Could they improve our understanding of storm dynamics? Johns Hopkins APL Tech. Dig., 21, 86-93.

,-- W. T. Liu, and P. G. Black, 2002: Microwave remote sensing of tropical cyclones from space. J. Oceanogr., 58, 137151.

Kazakov, A., and V. Lykosov, 1980: Parameterization of the interaction between the atmosphere and the surface in numerical modeling of atmospheric processes (in Russian). Proc. Numerical Methods of Weather Forecasting, East-Siberian Meteorological Regional Institute, 3-20.

Kepert, J., and Y. Wang, 2001: The dynamics of boundary layer jets within the tropical cyclone core. Part II: Nonlinear enhancement. J. Atmos. Sci., 58, 2485-2501.

Khain A. P., and L. K. Ingel, 1988: A numerical model of the atmospheric boundary layer above the ocean in the presence of convection. Atmos. Oceanic Phys., 24, 24-32.

$\longrightarrow$, and — 1995: Numerical modeling of interaction of a nonstationary divergent flow with convective processes in the boundary layer over the ocean. Atmos. Oceanic Phys., 31, 496506.

_ - M. G. Yarmolinskaya, and L. K. Ingel, 1986: Numerical modeling of interaction of convective and large-scale processes in the atmospheric boundary layer with the formation of a temperature inversion. Atmos. Oceanic Phys., 22, 987-993.

Kurihara, Y., and R. E. Tuleya, 1974: Structure of a tropical cyclone developed in a three-dimensional numerical simulation model. J. Atmos. Sci., 31, 893-919.

— - — , and M. A. Bender, 1998: The GFDL hurricane prediction system and its performance in the 1995 hurricane season. Mon. Wea. Rev., 126, 1306-1322.

LeMone, M. A., 1973: The structure and dynamics of horizontal roll vortices in the planetary boundary layer. J. Atmos. Sci., 30, 10771091.

__ 1976: Modulation of turbulence energy by longitudinal rolls in an unstable planetary boundary layer. J. Atmos. Sci., 33, 13081320 .

Liu, Q., and Y. Kogan, 1998: Large eddy simulations of cloud processing of small size aerosols in marine stratocumulus. Preprints, Conf. on Cloud Physics, Everett, WA, Amer. Meteor. Soc., 329332 .

Mellor, G. L., and T. Yamada, 1982: Development of a turbulence closure model for geophysical fluid problems. Rev. Geophys. Space Phys., 20, 851-875.

Moeng, C.-H., 1984: A large-eddy-simulation model for the study of planetary boundary-layer turbulence. J. Atmos. Sci., 41, 2052 2062.

_ - and J. C. Wyngaard, 1989: Evaluation of turbulent transport and dissipation closures in second-order modeling. J. Atmos. Sci., 46, 2311-2329.

- and P. P. Sullivan, 1994: A comparison of shear- and buoyancydriven planetary boundary layer flows. J. Atmos. Sci., 51, 9991022 .

Morrison, I., F. D. Marks, and S. Businger, 2002: WSR-88D observations of boundary layer rolls during hurricane landfall. Preprints, 25th Conf. on Hurricanes and Tropical Meteorology, San Diego, CA, Amer. Meteor. Soc., 341-342.

Pope, S. B., 2000: Turbulent Flows. Cambridge University Press, 771 pp.

Powell, M. D., P. J. Vickery, and T. A. Reinhold, 2003: Reduced drag coefficient for high wind speeds in tropical cyclones. Nature, 422, 279-283.

Randall, D., M. Khairoutdinov, A. Arakawa, and W. Grabowski, 2003: Breaking the cloud parameterization deadlock. Bull. Amer. Meteor. Soc., 84, 1547-1564.

Soong, S.-T., and Y. Ogura, 1980: Response of trade wind cumuli to large-scale processes. J. Atmos. Sci., 37, 2035-2050.

Tuleya, R. E., 1994: Tropical storm development and decay: Sensitivity to surface boundary conditions. Mon. Wea. Rev., 122, 291304.

Wurman, J., and J. Winslow, 1998: Intense sub-kilometer-scale boundary layer rolls observed in Hurricane Fran. Science, 280, 555-557. 